\title{
Why do free flap vessels thrombose? Lessons learned from implantable Doppler monitoring
}

\author{
Jason G Williams MD, Rodney J French MD FRCSC, Donald H Lalonde MD MSc FRCSC
}

JG Williams, RJ French, DH Lalonde. Why do free flap vessels thrombose? Lessons learned from implantable Doppler monitoring. Can J Plast Surg 2004;12(1):23-26.

BACKGROUND: Before implantable venous Doppler monitoring, by the time the failing flap was explored, thrombosis had often occurred and therefore the cause of flap flow cessation was often difficult to determine. The Doppler allowed the detection of flow cessation in failing flaps before thrombosis occurred in every case since the authors started using it in 1999.

OBJECTIVES: To review the authors' experiences with the implantable venous Doppler.

METHODS: The authors reviewed 43 free flaps in 40 consecutive patients (1999 to 2002) in which the implantable venous Doppler was used. All cases were performed at the Saint John Regional Hospital, Saint John, New Brunswick, by the senior author. Data were collected from the hospital and office charts.

RESULTS: The Doppler detected inadequate blood flow in nine free flaps. In five of the cases, the cause was a kink in the vein. Repositioning the vein to get rid of the kink salvaged all five flaps. In the sixth case, compression of the vein after insetting was detected and successfully corrected. Flow cessation in the seventh case was attributable to arterial vasospasm, which was also salvaged. In the last two cases, the cause was low flow in the flap from the time the vessel clamps were let go. In spite of patent anastomoses, these two flaps were lost because there was not enough flow to sustain them.

CONCLUSION: The implantable venous Doppler has allowed intraoperative detection of free flap vessel flow cessation, identification of the reasons for, and the correction of these prethrombotic states.

Key Words: Free flap; Implantable Doppler; Thrombosis

\section{Le Doppler implantable révèle les causes des thromboses vasculaires dans les lambeaux libres}

HISTORIQUE : Avant l'avènement du Doppler implantable, la thrombose se produisait souvent avant que l'on puisse même examiner le lambeau défaillant. Il était par conséquent difficile de déterminer la cause de l'arrêt de la circulation dans le lambeau. À chaque fois depuis que les auteurs ont commencer à l'utiliser, en 1999, le Doppler a permis de détecter l'arrêt de la circulation sanguine dans les lambeaux défectueux avant la formation d'une thrombose.

OBJECTIF : Faire le point sur l'expérience acquise par les auteurs avec le Doppler veineux implantable.

MÉTHODE : Les auteurs ont passé en revue 43 lambeaux pratiqués chez 40 patients consécutifs (de 1999 à 2002) et pour lesquels le Doppler veineux implantable a été utilisé. Toutes ces chirurgies ont été effectuées à l'Hôpital Régional de Saint-Jean, Nouveau-Brunswick, par l'auteur principal. Les données ont été colligées à partir des dossiers des hôpitaux et des cabinets médicaux.

RÉSULTATS : Le Doppler a permis la détection de problèmes de circulation sanguine dans neufs lambeaux libres. Dans cinq cas, la veine était étranglée par un vrillage. Les cinq lambeaux ont pu être sauvés par un repositionnement de la veine. Dans le sixième cas, on a détecté une compression de la veine après l'insertion et la situation a pu être corrigée. Dans le septième cas, l'arrêt de la circulation était dû à un vasospasme artériel qui a également pu être corrigé. Les deux derniers cas avaient pour cause un faible débit veineux dans le lambeau après le retrait des clamps. Malgré la perméabilité des anastomoses, ces deux lambeaux n'ont pas pu être récupérés en raison de l'irrigation sanguine insuffisante.

CONCLUSION : Le Doppler veineux implantable a permis le dépistage peropératoire des blocages circulatoires dans les lambeaux libres, l'identification de leur cause et la correction de ces états préthrombotiques.
$\mathrm{M}$ aintenance of patent vascular anastomoses is fundamental to the success of any free tissue transfer. The art and science of microsurgery has progressed steadily over the past 30 years, with advances in technical skills and equipment, and the discovery of more reliable flaps. Free flap success rates have risen from $94 \%$ in a 1984 survey to $98.8 \%$ in 1992 $(1,2)$. Salvage rates for failing free flaps have not increased to the same degree. It is suggested by several authors that this is a result of late detection of vascular compromise in the flap $(2-5)$.

Thrombosis of the vascular pedicle has been the focus of much attention in effort to reduce its incidence through technical and pharmacological means. The classic causes of thrombosis described by Virchow in his famous triad are well known. Stasis, hypercoagulability and endothelial damage lead to thrombus formation. Less well recognized are the factors that create a prethrombotic state. For free flaps specifically, Khouri (1) outlines factors suspected of leading to thrombosis as being kinks or twists in the pedicle, tight closure or edema, hematoma, and spasm or inadequate flow.

Clinical evaluation remains the gold standard to which all other methods of monitoring must be compared. This includes observing skin colour, temperature, capillary refill and pinprick testing $(5,7,8)$. However, as demonstrated by Swartz $(3,4)$ in his work on this subject, cessation of flow from venous obstruction can occur hours before development of clinical signs of

Division of Plastic Surgery, Dalhousie University, Saint John Campus, Saint John, New Brunswick

Correspondence and reprints: Dr Donald H Lalonde, Division of Plastic Surgery, Saint John Regional Hospital, 400 University Avenue,

Saint John, New Brunswick E2L 4L2. Telephone 506-648-7950, fax 506-652-8042, e-mail huzil@health.nb.ca

Presented at the 57th Annual Meeting of the Canadian Society of Plastic Surgery, June 25-29, 2003, Whistler, British Columbia 


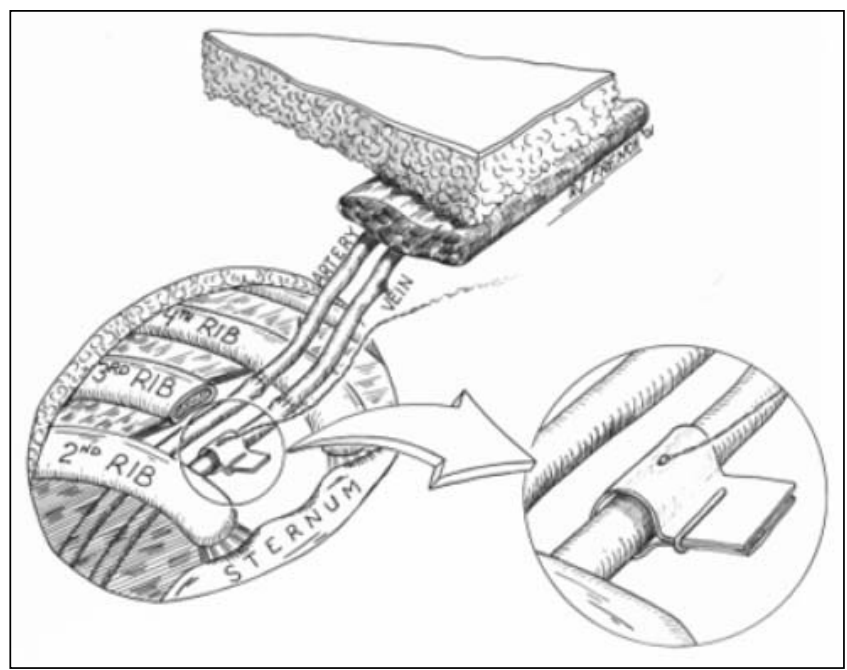

Figure 1) Illustration of a free transverse rectus abdominis muscle flap with the Cook-Swartz Doppler Flow Probe attached to the internal mammary vein, downstream of the microvascular anastomosis. It is secured to the vein with a silastic cuff (inset)

venous congestion, and arterial flow may persist for a variable period of time in the presence of venous obstruction $(3,4)$.

The Cook-Swartz implantable venous Doppler provides real-time, continuous feedback on the status of the vascular pedicle, early detection of failing flaps and a better salvage rate $(2-4,8)$. Its usefulness in postoperative free flap monitoring has been demonstrated in several studies, including an earlier assessment of our centre's experience by French et al (8) in 2001. The implantable Doppler has been particularly effective in the monitoring of buried flaps, where clinical monitoring is not an option. Kind et al (2), in a 1998 review of the Buncke Clinic experience, reported a significantly improved free flap salvage rate, from a pre-Doppler rate of $71 \%$ to $100 \%$ with Doppler monitor use.

The utility of the implantable Doppler for intraoperative monitoring has been alluded to in previous articles $(2,3,8)$, but has not been specifically studied. It is suggested that this period is actually more critical than the postoperative monitoring period in early detection of vascular compromise $(2,3,8)$.

We found the implanted venous Doppler probe to be an invaluable tool during free flap surgery. In this paper we review our experience with this device, specifically examining those cases where blood flow problems were detected, the cause identified, and corrected intraoperatively.

\section{Design}

\section{METHODS}

A retrospective review of all patients receiving free flaps with implantable venous Doppler monitoring between 1999 and 2002 at the Saint John Regional Hospital, Saint John, New Brunswick, was performed.

\section{Data acquisition}

Data collected included patient demographics, flap type and destination, ischemia time, hospital stay, and details surrounding any loss of venous Doppler signal. Data were obtained from hospital records and office charts.

\section{Technique}

Following completion of the microvascular anastomosis, the $20 \mathrm{MHz}$ Cook-Swartz Doppler flow probe (model DP-SDP001, Cook Vascular Inc, USA) was fixed to the recipient vein downstream of the anastomosis with a silastic cuff (Figure 1). The cuff was maintained in place with a small vascular clip. The transducing wire was attached to the bedside monitor, and position was confirmed by hearing the characteristic venous flow. This sound has been described as the sound of the ebb and flow of the ocean or the howl of the wind. Manual pressure on the flap, like pressing on a sponge, created a rush of venous blood across the Doppler probe and resulted in the previously described 'whoosh sign'. The Valsalva maneuver decreased venous flow in the flap vein and resulted in a diminished venous signal from the Doppler called the 'heave sign'. The monitor was left on for the remainder of the surgery and for three to five days postoperatively. Nursing staff involved in postoperative care were educated regarding the monitoring sound, and instructed to contact the surgeon if any change in sound occured.

\section{RESULTS}

Over the three-year period spanned by the present study, 43 flaps in 40 patients were performed and monitored with the implanted venous Doppler. Three patients had bilateral free flap breast reconstruction. There were two flap failures, for an overall success rate of $95 \%$. Demographic details are listed in Table 1 . A summary of the types of flaps used and indications is presented in Tables 2 and 3.

Of the nine flow problems detected by the venous Doppler, seven were complete signal losses and two were poor quality, low flow states. Table 4 summarizes the seven cases of intraoperative signal loss $(16 \%)$, that were found to be a result of pedicle kink, venous compression and arterial spasm. Detecting these problems intraoperatively permitted exploration of the pedicles immediately, restoring flow to the flap at the earliest possible time, which is critical to successful salvage.

Two further cases were identified as low flow flaps. Despite patent anastomoses in both of these cases, there was inadequate flow to perfuse the flaps. The Doppler signal was consistently of poor quality in these cases, prompting exploration and revision of anastomoses. Adequate flow was not possible, and the flaps were ultimately lost, leading to a failure rate of $4.7 \%$. These losses, in hindsight, were foretold by the poor Doppler signal.

There were four cases that were taken back to the operating room for exploration (take back rate 9.3\%). One of these was a low flow flap, detected at the initial operation. This flap still had patent anastomoses, but was not salvageable because of the low flow. Sound was lost postoperatively in one case, and on re-exploration it was found that the probe had become detached from the vessel. It was reattached and the flap survived. Another flap appeared dusky clinically despite good Doppler signal. On exploration, the vessels were found to be patent, no revision was required, and the flap survived. There was one case of early postoperative thrombosis at the anastomosis, which represents a tenth case of flow cessation detected by the Doppler. The patient was taken back to the operating room, the anastomosis was revised and the flap survived.

The Doppler probe remained in place an average of 4.1 days. In each case the probe was removed with gentle traction on the lead wire, which delivered it from the wound. 
TABLE 1

Demographics of patients undergoing free flap reconstruction at the Saint John Regional Hospital between 1999 and 2002

\begin{tabular}{ll}
\hline Average age (years) & 50.6 \\
Age range (years) & $18-81$ \\
Females & 36 \\
Males & 4 \\
\hline
\end{tabular}

TABLE 2

Types of free flaps performed between 1999 and 2002 at Saint John Regional Hospital

\begin{tabular}{lc}
\hline Flap & Frequency \\
\hline Free TRAM & 15 \\
Perforator TRAM (DIEP) & 19 \\
Rectus abdominis & 4 \\
Fibula & 2 \\
Latissimus dorsi & 2 \\
Radial forearm & 1 \\
Total & 43 \\
\hline
\end{tabular}

DIEP Deep inferior epigastric perforator; TRAM Transverse rectus abdominis muscle

There were no complications associated with probe removal. The average length of hospital stay was 7.8 days.

\section{Representative cases}

Case 1: A 55-year-old man with a history of ethanol abuse, smoking and diet-controlled diabetes had a motorcycle accident many years prior and had undergone eight previous operations for tibial nonunion. He presented for debridement of infected bone and reconstruction with a free rectus abdominis muscle flap. Uncomplicated microvascular anastomoses were performed and the Doppler was applied with good initial flow. During insetting and suturing of flap the Doppler went silent. Sound returned instantaneously with release of sutures. Both anastomoses were patent on examination. Subsequent attempts at insetting also caused loss of Doppler sound secondary to compression of the vein. Finally, the flap was loosely inset to maintain flow to the muscle. The Doppler remained audible throughout the postoperative period and the flap survived with no further problems. The Doppler clearly told us that we could not firmly inset this muscle flap.

Case 2: A 55-year-old woman with a history of previous mastectomy underwent delayed left breast reconstruction with a free transverse rectus abdominis muscle flap. The microvascular anastomoses went smoothly and the Doppler was applied with excellent flow signal. As the last skin suture was placed, the Doppler went silent. The flap was raised medially, and the vein was found to be kinked. The vein was un-kinked and the flow signal returned immediately. The Doppler remained audible throughout the postoperative period, and the flap survived.

\section{DISCUSSION}

In the present study we found that kinking of the vascular pedicle was the most common cause of free flap vessel occlusion. Mechanical occlusion of the vascular pedicle created a prethrombotic state that, if not corrected early, can lead to thrombosis and irreversible flap damage. Through continuous monitoring provided by the implantable venous Doppler, we were able to
TABLE 3

Flap destination

\begin{tabular}{lc}
\hline Destination of flap & Frequency \\
\hline Mastectomy defect & 33 \\
Head and neck & 3 \\
Lower limb & 6 \\
Upper limb & 1
\end{tabular}

TABLE 4

Causes of flow disturbance detected intraoperatively with the venous Doppler flow probe

\begin{tabular}{lcc}
\hline Cause of flow disturbance & Incidence & Number salvaged \\
\hline Pedicle kink & 5 & 5 \\
Pedicle compression & 1 & 1 \\
Vasospasm & 1 & 1 \\
Low flow & 2 & 0 \\
\hline
\end{tabular}

detect these problems and correct them at the earliest possible time, usually before the patient left the operating room.

As demonstrated by Kerrigan et al (6), failing free flaps have a higher salvage rate if detected early. They found that there is far less tolerance of tissue to secondary ischemia than to primary ischemia. Secondary ischemia was defined as the time elapsed between loss of flap circulation to successful restoration of flow. It was found that $50 \%$ of flaps did not survive after 7.2 hours of secondary ischemia, while they would withstand 13.2 hours of primary ischemia. These findings underline the importance of prompt recognition of free flap failure, and urgent re-exploration. Because the venous Doppler signal will be lost before the arterial pulse and before clinical manifestations of venous congestion in cases of circulatory failure (3), the venous Doppler flow probe can and does play an important role in early detection of flap failure. Indeed, in our experience, flow occlusion and subsequent flap ischemia was detected instantaneously, minimizing secondary ischemic insult.

Khouri (1) outlined factors suspected of leading to thrombosis as being kinks or twists of the pedicle, tight closure or edema, hematoma, and spasm or inadequate flow. Hidalgo and Jones (9) found that seven of 11 patients who had flap reexploration in their series of 150 free flaps clearly had venous problems, such as a pedicle kink or external compression. As with our population, no patient had primary anastomotic thrombosis present at time of re-exploration. These mechanical factors interrupt perfusion of the free flap. Heller and Levin (7) elegantly described the effects of venous flow occlusion:

"Venous insufficiency can occur due to torsion of the pedicle, flap edema, hematoma, or tight closure of the tissue over the pedicle. Venous outflow obstruction can result in extravasation of the red blood cells, endothelial breakdown, thrombosis in the microcirculation, and flap death. Given the irreversible nature of the microcirculatory changes in venous congestion that occur even after short periods of time, the surgeon must recognize venous compromise as early as possible."

Our two flap failures were attributed to inadequate flow, despite patent anastomoses. The Doppler signal was poor from the time of clamp release, and in hindsight this predicted later problems. Swartz et al (4) recognized the significance of poor 
signals in the operating room, commenting that these are indicators of probable troubles to come.

Because pedicle kinks have such significant consequences, it is worthwhile considering why pedicles become kinked. In the period after the anastomoses are completed, the flap vessels gradually open up and venous outflow increases. This causes the pedicle vein to fill and it becomes fatter and longer. We have observed that gentle curves in the pedicle can become kinks as the vein elongates. The kinks cause mechanical obstruction of flow, creating stasis. As we know, stasis leads to thrombosis therefore a kinked vessel is in a prethrombotic state.

Detection and correction of these problems during the initial surgery probably prevented early flap compromise and urgent return to the operating room, with the costs to the patient, surgeon, and system associated with it.

\section{What have we learned from this experience?}

- Most of our vascular occlusions occurred during wound closure;

- Most of our vascular occlusions were caused by kinks in the expanding pedicle; and

- The buried venous Doppler allowed immediate detection of kinks and enabled us to unkink the pedicle before venous thrombosis occurred.

There are limitations of this study. It cannot be stated with complete certainty that the flaps whose Doppler signal was lost intraoperatively would have become clinically compromised postoperatively. With repositioning and hemodynamic changes, for example, some of the causes of flow cessation may have resolved. This is also a retrospective review, and shares all the faults of this study design.

\section{CONCLUSION}

In our experience, we have found that compromise of free flap blood flow is usually caused by mechanical obstruction, most often in the form of a kinked pedicle. The implantable Doppler probe has provided a window on the free flap vascular pedicle that we have taken advantage of for intra-operative monitoring and detection flow interruption. We now understand why our pedicles may ultimately thrombose, and have the ability to detect and correct these prethrombotic states during the original surgery.

\section{REFERENCES}

1. Khouri RK. Avoiding free flap failure. Clin Plast Surg 1992;19:773-81.

2. Kind GM, Buntic RF, Buncke GM, Cooper TM, Siko PP, Buncke HJ. The effect of an implantable Doppler probe on the salvage of microvascular tissue transplants. Plast Reconstr Surg 1998;101:1268-73.

3. Swartz WM, Jones NF, Cherup L, Klein A. Direct monitoring of microvascular anastomoses with the 20- $\mathrm{MHz}$ ultrasonic Doppler probe: An experimental and clinical study. Plast Reconstr Surg 1988;81:149-61.

4. Swartz WM, Izquierdo R, Miller MJ. Implantable venous Doppler microvascular monitoring: Laboratory investigation and clinical results. Plast Reconstr Surg 1994;93:152-63.

5. Jones NF. Intraoperative and postoperative monitoring of microsurgical free tissue transfers. Clin Plast Surg 1992;19:783-97.

6. Kerrigan CL, Zelt RG, Daniel RK. Secondary critical ischemia time of experimental skin flaps. Plast Reconstr Surg 1984;74:522-6.

7. Heller L, Levin LS. Lower extremity microsurgical reconstruction. Plast Reconstr Surg 2001;108:1029-41.

8. French RJ, O'Brien JP, Sparkes GL, Lalonde DH. Free flap monitoring using an implantable Doppler probe. Can J Plast Surg 2001;9:233-8.

9. Hidalgo DA, Jones CS. The role of emergent exploration in free tissue transfer: A review of 150 consecutive cases. Plast Reconstr Surg 1990;86:492-8. 